Commun. Korean Math. Soc. 24 (2009), No. 4, pp. 561-564

DOI 10.4134/CKMS.2009.24.4.561

\title{
ON A BESOV SPACE AND RADIAL LIMITS
}

\author{
Pil Lan Kim, Ern Gun Kwon, and Jong Hee Park
}

Abstract. A holomorphic function space in the unit disc $D$ satisfying

$$
\int_{D}\left|f^{\prime}(z)\right|^{p}\left(1-|z|^{2}\right)^{p-1} d A(z)<\infty
$$

is quite close to $H^{p}$. The problems on the existence of the radial limits are considered for this space. It is proved that the situation for $p>2$ is totally different from the situation for $p \leq 2$.

\section{Introduction}

Let $A B_{p, p+1}$ denote the Besov space consisting of holomorphic $f$ in the unit disc $D$ of he complex plane for which

$$
\int_{D}\left|f^{\prime}(z)\right|^{p}\left(1-|z|^{2}\right)^{p-1} d A(z)<\infty .
$$

Here and throughout $d A(z)=d x d y, z=x+i y$.

We, in this paper, consider the existence of the radial limits of the functions in $A B_{p, p+1}$. First, if $0<p \leq 2$, then by a well-known theorem [4, Theorem XIV(3.24)], we have $A B_{p, p+1} \subset H^{p}$, where $H^{p}$ denotes the classical Hardy space which consists of holomorphic $f$ in $D$ satisfying

$$
\|f\|_{p}:=\left(\int_{0}^{2 \pi}\left|f\left(r e^{i \theta}\right)\right|^{p} \frac{d \theta}{2 \pi}\right)^{1 / p}<\infty .
$$

Hence $f$ should have radial limits almost everywhere on $T=\partial D$.

Our question is "What about $p>2$ ?" The answer to the question (and a similar question for $\mathbb{R}_{+}^{n+1}$ ) might be known to experts. We settle down the problem in this paper by a simple method.

Theorem 1.1. Let $2<p<\infty$. Then there is $f \in A B_{p, p+1}$ such that $f$ has radial limits almost nowhere on $T$.

So, the situation for $p>2$ is totally different from the situation for $p \leq 2$. Moreover, we have:

Received April 10, 2009.

2000 Mathematics Subject Classification. Primary 30H05.

Key words and phrases. radial limits, Besov space.

This work was supported by Andong National University 2006.

(C)2009 The Korean Mathematical Society 
Corollary 1.2. Let $0<p<\infty$. Then the space $A B_{p, p+1}$ belongs to the Nevanlinna class if and only if $0<p \leq 2$.

See $[1,4]$ for the Nevanlinna class and $H^{p}$. Corollary 1.2 follows directly from Theorem 1.1 and our proof of Theorem 1.1 is constructive one using several known facts of the next section.

\section{Preliminaries}

For a function $f$ holomorphic in $D$ and for $0 \leq r<1$, we denote

$$
M_{p}(r, f)=\left(\int_{0}^{2 \pi}\left|f\left(r e^{i \theta}\right)\right|^{p} \frac{d \theta}{2 \pi}\right)^{1 / p}
$$

if $0<p<\infty$ and

$$
M_{\infty}(r, f)=\sup _{0 \leq \theta \leq 2 \pi}\left|f\left(r e^{i \theta}\right)\right| .
$$

For $0<p \leq \infty, 0<q<\infty$ and $-1<\alpha<\infty, A^{p, q, \alpha}$ denotes the mixed normed space consisting of holomorphic $f$ in $D$ for which

$$
\int_{0}^{1}(1-r)^{\alpha} M_{q}(r, f)^{p} d r<\infty .
$$

For $0<q, r \leq \infty$, we denote by $\ell(q, r)$ the set of those sequences $\left\{a_{k}\right\}_{k=0}^{\infty}$ for which

$$
\left\{\left(\sum_{k \in I_{m}}\left|a_{k}\right|^{q}\right)^{1 / q}\right\} \in \ell^{r} \quad(q<\infty)
$$

and

$$
\left\{\sup _{k \in I_{m}}\left|a_{k}\right|\right\} \in \ell^{r} \quad(q=\infty),
$$

where

$$
I_{m}=\left\{k: 2^{m} \leq k<2^{m+1}\right\} \quad(m=1,2, \ldots)
$$

and $I_{0}=\{0\} \cdot \ell(q, r)$ forms a normed linear space if $1 \leq q, r \leq \infty$. For dual spaces and multipliers between these spaces we refer to [2].

Note that if $f(z)=\sum_{0}^{\infty} a_{k} z^{k}$ is holomorphic in $D$, then $f$ can be set in oneto-one correspondence with the sequence $\left\{a_{k}\right\}_{k=0}^{\infty}$. We identify a holomorphic function with the sequence of its Taylor coefficients.

Theorem 2.1 ([1]). Let $a_{1}, a_{2}, \ldots$ be complex numbers such that

$$
\limsup _{n \rightarrow \infty}\left|a_{n}\right|^{1 / n}=1 \text {. }
$$

(i) If $\sum\left|a_{n}\right|^{2}<\infty$, then for almost every choice of sign $\left\{\epsilon_{n}\right\}$,

$$
f(z)=\sum_{n=0}^{\infty} \epsilon_{n} a_{n} z^{n} \in H^{p} \quad \text { for all } p<\infty .
$$

(ii) If $\sum\left|a_{n}\right|^{2}=\infty$, then for almost every choice of sign $\left\{\epsilon_{n}\right\}, f(z)$ has a radial limit almost nowhere. 
Theorem $2.2([3])$. For $0<p<\infty, 1 \leq q \leq 2$ and $-1<\alpha<\infty$,

$$
A^{p, q, \alpha} \subset I^{-(\alpha+1) / p} \ell\left(q^{\prime}, p\right),
$$

where $q^{\prime}$ is the conjugate exponent of $q$.

(2.1) means that if $f(z)=\sum_{0}^{\infty} a_{n} z^{n} \in A^{p, q, \alpha}$, then $\left\{n^{(\alpha+1) / p} a_{n}\right\}_{0}^{\infty} \in \ell\left(q^{\prime}, p\right)$. Of course we have an inclusion reverse to (2.1) by duality.

Theorem 2.3. For $0<p<\infty, 2 \leq q \leq \infty$ and $-1<\alpha<\infty$,

$$
I^{-(\alpha+1) / p} \ell\left(q^{\prime}, p\right) \subset A^{p, q, \alpha},
$$

where $q^{\prime}$ is the conjugate exponent of $q$.

\section{Proof of Theorem 1.1}

For a sequence $\epsilon=\left\{\epsilon_{k}= \pm 1\right\}$, take

$$
f_{\epsilon}(z)=\sum_{k=1}^{\infty} \frac{\epsilon_{k}}{\sqrt{k}} z^{2^{k}}, \quad z \in D .
$$

Then we have by the harmonic series divergence

$$
\sum\left|\frac{\epsilon_{k}}{\sqrt{k}}\right|^{2}=\sum\left|\frac{1}{\sqrt{k}}\right|^{2}=\infty
$$

Since

$$
\limsup _{k \rightarrow \infty}\left|\frac{1}{\sqrt{k}}\right|^{\frac{1}{2^{k}}}=\lim _{k \rightarrow \infty} \frac{1}{k^{\frac{1}{2} \frac{1}{2^{k}}}}=\frac{1}{e^{\lim _{k \rightarrow \infty} \frac{1}{2^{k+1}} \ln k}},
$$

we obtain also

$$
\limsup _{k \rightarrow \infty}\left|\frac{\epsilon_{k}}{\sqrt{k}}\right|^{\frac{1}{2^{k}}}=1
$$

Thus, by Theorem 2.1, almost every choice of signs $\epsilon=\left\{\epsilon_{k}\right\}, f_{\epsilon}(z)$ has a radial limit almost nowhere.

But we are going to show

$$
\int_{D}\left|f_{\epsilon}^{\prime}(z)\right|^{p}\left(1-|z|^{2}\right)^{p-1} d A<\infty
$$

for $p>2$, which gives what we want.

In order to verify (3.1), first note that (3.1) is nothing but $f_{\epsilon} \in I A^{p, p, p-1}$. In view of $(2.2)$ we are sufficient to prove that

$$
f_{\epsilon} \in l\left(p^{\prime}, p\right) \text {. }
$$

Since $f_{\epsilon}$ is a gap sequence with

$$
\sum \frac{1}{(\sqrt{k})^{p}}<\infty
$$

for $p>2$, it follows that $f_{\epsilon}$ satisfies (3.2). The proof is complete. 


\section{Further question}

Of course, there may be many ways to approach the problem on radial limits. Refining the Besov spaces we considered, we pose a further question: Characterize all the radial function $\omega(z)$ such that there is a holomorphic function $f$ defined on $D$ for which

$$
\int_{D}\left|f^{\prime}(z)\right|^{p} \omega(z) \frac{d A(z)}{1-|z|^{2}}<\infty
$$

but has radial limits almost nowhere on $T$.

\section{References}

[1] P. L. Duren, Theory of $H^{p}$ Spaces, Academic Press, New York, 1970.

[2] C. N. Kellog, An extension of the Hausdorff-Young theorem, Michigan Math. J. 18 (1971), 121-127.

[3] E. G. Kwon, A note on the coefficients of mixed normed spaces, Bull. Austral. Math. Soc. 33 (1986), 253-260.

[4] A. Zygmund, Trigonometric Series, Cambridge University Press, London, 1959.

Pil LAN Kim

Department of Mathematics Education

Andong National University

ANDONG 760-749, KOREA

E-mail address: pillan119@hanmail.net

ERn Gun KwON

Department of Mathematics Education

Andong National University

ANDONG 760-749, Korea

E-mail address: egkwon@andong.ac.kr

Jong HeE PARK

Department of Mathematics Education

Andong National University

ANDONG 760-749, KoreA

E-mail address: jh0021@hanmail.net 